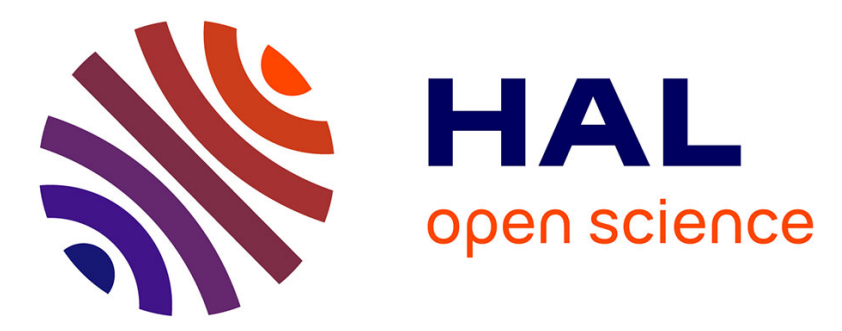

\title{
Integrated simulation of ground motion mitigation, techniques for the future compact linear collider (CLIC)
}

\author{
G. Balik, B. Caron, D. Schulte, J. Snuverink, J. Pfingstner
}

\section{To cite this version:}

G. Balik, B. Caron, D. Schulte, J. Snuverink, J. Pfingstner. Integrated simulation of ground motion mitigation, techniques for the future compact linear collider (CLIC). Nuclear Instruments and Methods in Physics Research Section A: Accelerators, Spectrometers, Detectors and Associated Equipment, 2013, 700, pp.163-170. 10.1016/j.nima.2012.10.031 . in2p3-00743493

HAL Id: in2p3-00743493

https://hal.in2p3.fr/in2p3-00743493

Submitted on 1 Oct 2013

HAL is a multi-disciplinary open access archive for the deposit and dissemination of scientific research documents, whether they are published or not. The documents may come from teaching and research institutions in France or abroad, or from public or private research centers.
L'archive ouverte pluridisciplinaire HAL, est destinée au dépôt et à la diffusion de documents scientifiques de niveau recherche, publiés ou non, émanant des établissements d'enseignement et de recherche français ou étrangers, des laboratoires publics ou privés. 


\title{
Integrated simulation of ground motion mitigation techniques for the future compact linear collider (CLIC)
}

\author{
G. Balik ${ }^{1}$, B. Caron ${ }^{2}$, D. Schulte ${ }^{3}$, J. Snuverink ${ }^{3,4}$, J. Pfingstner ${ }^{3}$ \\ ${ }^{1}$ : LAPP-IN2P3-CNRS - Université de Savoie - Annecy-le-Vieux, France \\ 2: SYMME-Polytech'Annecy Chambéry - Université de Savoie - Annecy-le-Vieux, France \\ ${ }^{3}$ : CERN-European Organization for Nuclear Research, Geneva, Switzerland \\ ${ }^{4}:$ John Adams Institute at Royal Holloway, Egham, Surrey, UK
}

ARTICLE INFO

Article history:

Keywords:

Trajectory control

Vibration rejection

Nano-control

Beam-based feedbacks

Beam dynamic simulation

\section{ABSTRACT}

CLIC is a proposal of CERN for a future high-energy particle collider. CLIC will collide electron and positron beams at a centre of mass energy of $3 \mathrm{TeV}$ with a desired peak luminosity of $2 * 10^{34} \mathrm{~cm}^{-2} \mathrm{~s}^{-1}$. The luminosity performance of CLIC is sensitive to ground motion. Ground motion misaligns accelerator components, most importantly quadrupole magnets, which leads to emittance growth and beam-beam offset at the interaction point. This paper discusses the beam based feedback strategies currently used together with mechanical stabilization systems to address the above mentioned issues. These strategies consist of an Interaction Point Feedback (IPFB) and an Orbit Feedback (OFB). The two feedbacks have been designed independently and the main objective of this paper is to show how they interact. A simulation program is used in order to simulate the whole collider, it includes the behaviour of the beams, magnets, supports, ground attenuators, sensors, and actuators. Beamoffset feedback optimization and integrated simulations have been performed and results show that despite a detrimental coupling of both feedbacks at high frequency, it is possible to decrease the beam-beam offset and maintain the desired luminosity. 


\section{Introduction}

Future linear colliders could open the way to new exciting physics. One design is the Compact Linear Collider (CLIC) [1]. This particle accelerator is expected to answer some of the fundamental questions regarding the nature of matter. The feasibility study of such an ambitious project is carried out by an international collaboration of scientists. Among its numerous technical challenges, CLIC needs unprecedented Ground Motion (GM) mitigation techniques. These techniques, composed of mechanical stabilization systems and Beam-Based Feedbacks (BBFB) are necessary to achieve the desired performances in terms of luminosity: $L_{0}=2 * 10^{34} \mathrm{~cm}^{-2} \mathrm{~s}^{-1}$ [1]. Luminosity and the rate of physics events is correlated to the beam emittances (linked to the beam size) and the relative beam-beam offset at the Interaction Point (IP) according to equation 1 , where $L$ is the obtained luminosity, $x$ and $y$ are the vertical and horizontal directions, $\sigma_{(0)}$ the (nominal) beam size at the IP and $\delta$ the beam-beam offset at the IP along $x$ and $y$. Note that the equation is an approximation and only valid for small deviations around the nominal values $\left(\sigma_{x, 0}=45 \mathrm{~nm}\right.$ and $\left.\sigma_{y, 0}=1 \mathrm{~nm},[1]\right)$. As the vertical dimension of the beam is 45 times smaller than the horizontal one, only the former has been considered.

$$
\frac{L}{L_{0}} \approx \frac{e^{-\frac{1}{4}\left(\left(\frac{\delta_{x}}{\sigma_{x}}\right)^{2}+\left(\frac{\delta_{y}}{\sigma_{y}}\right)^{2}\right)}}{\frac{\sigma_{x} \sigma_{y}}{\sigma_{x, 0} \sigma_{y, 0}}}
$$

The CLIC luminosity loss budget due to all dynamic imperfections is about $20 \%$ [1]. Our goal is to have a small contribution from GM on the luminosity loss. A contribution of only $1 \%$ needs a vertical offset $\delta_{y}$ of $0.2 \mathrm{~nm}$ $\operatorname{IRMS}(0.1)$ (Integrated Root Mean Square at $0.1 \mathrm{~Hz}$ ). The IRMS is defined as the integral of the Power Spectral Density (PSD) within a given frequency range:

$$
\operatorname{IRMS}\left(f_{0}\right)=\int_{f_{0}}^{\infty} \quad P S D(f) d f
$$

This paper describes the two feedback controls currently used within the simulations. On the one hand, an optimized IPFB control localized at the IP uses the beam deflection angle, post-collision Beam Position Monitor (BPM) and a dedicated control to minimize the beam-beam offset thanks to kickers near the IP. On the other hand, the ultra low beam emittance preservation is facilitated by an Orbit Feedback (OFB). It uses the beam position all along the beam line obtained by BPMs in order to correct the beam oscillations. Integrated simulations of the beam line and the IP collisions, 
which includes both feedback controls studied in this paper, realistic modelling of the GM and technical noise and realistic transfer through supports (including mechanical feedback) have been performed by using PLACET [2], a tracking code developed at CERN, and the beam-beam code GUINEA-PIG [3]. Although being complementary, the simultaneous use of both feedbacks has some side effects leading to high frequency beam motion amplification. The first part of this paper describes the collider and the disturbances that affect the beam position and orbit. The second part is dedicated to the presentation of the two feedbacks that have been designed independently. The last part investigates the interaction between both feedbacks and shows that the desired performances can be reached after a re-optimization of the parameters.

\section{The Compact Linear Collider}

CLIC is an electron-positron collider with a nominal centre of mass energy of $3 \mathrm{TeV}$ with a peak luminosity of $2 * 10^{34}$ $\mathrm{cm}^{-2} \mathrm{~s}^{-1}[1]$. Fig. 1 gives an overall layout of the complex (taken from [1])

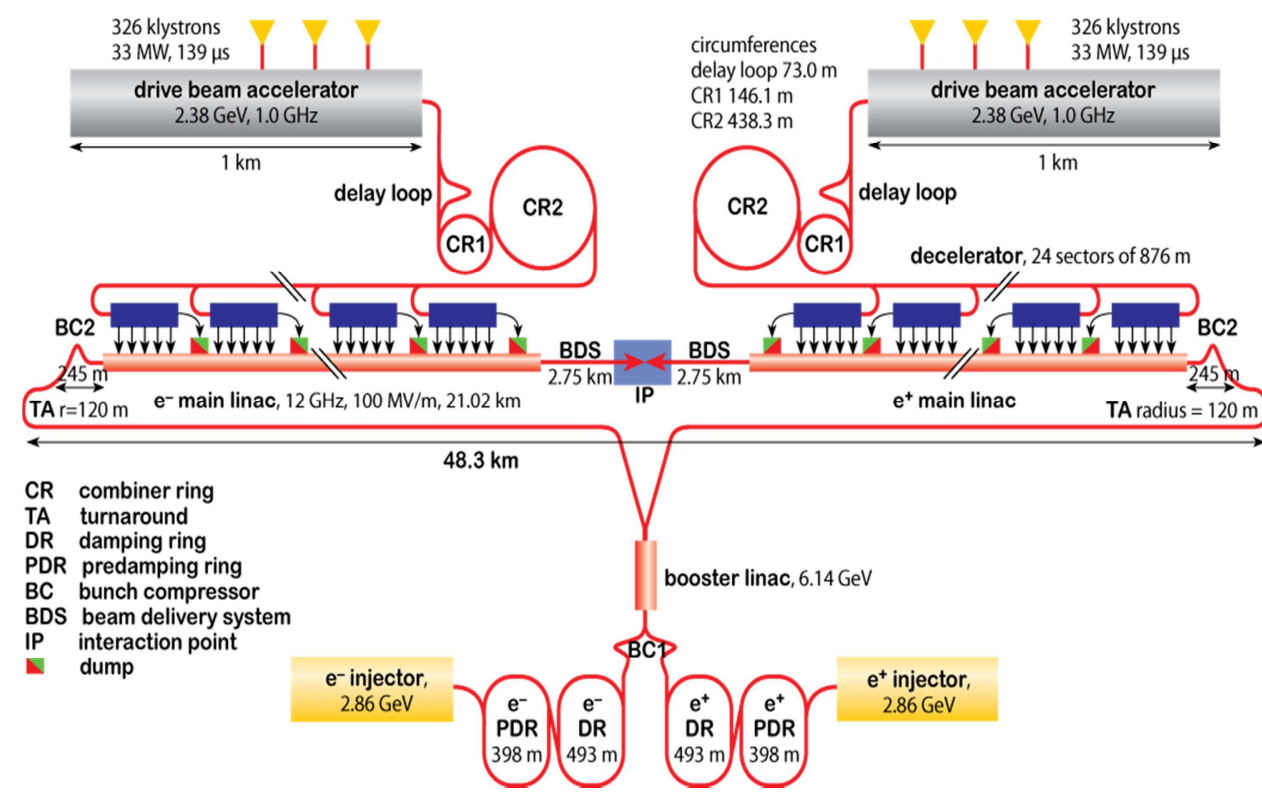

Fig. 1: Layout of the CLIC complex.

CLIC uses a novel two beam acceleration scheme to generate a high accelerating gradient of $100 \mathrm{MV} / \mathrm{m}$. This acceleration scheme together with an optimization for wall plug power efficiency results in short bunch intervals of $0.5 \mathrm{~ns}$ and a train length of 312 bunches of about $3.7 * 10^{9}$ particles each. A repetition rate of $50 \mathrm{~Hz}$ was chosen to synchronise the Radio Frequency (RF) power source with the wall plug network. The high luminosity requires very small beam emittance, which is generated in the damping rings. 


\subsection{CLIC beam line}

\subsubsection{Main linac}

The two main linacs (ML), one for positrons and the other for electrons, accelerate the beams from an initial energy of 9 $\mathrm{GeV}$ to the final value of $1.5 \mathrm{TeV}$ by using normal conducting accelerating structures with an $\mathrm{RF}$ of $12 \mathrm{GHz}$ and a gradient of $100 \mathrm{MV} / \mathrm{m}$. This choice of frequency and gradient is based on an optimization of the total accelerator cost. The ML design is identical for electrons and positrons and both MLs are about $21 \mathrm{~km}$ long. The key design goal is the preservation of the ultra-low transverse emittance during beam transport. This goal is achieved by a combination of careful lattice design, precise pre-alignment of the beam line components, stabilisation of the beam guiding quadrupoles against vibrations and by using beam-based correction methods. The ML tunnel and the beam line are laser straight [4].

\subsubsection{Beam Delivery System}

The Beam Delivery System (BDS) squeezes the beams and brings them into collision. Furthermore, the BDS should protect the beam line and the detector against missteered beams coming from the ML and remove beam-halo particles to minimise background in the detectors. This is performed by the collimation system. The first collimator needs to survive the impact of a complete bunch train, which requires large beam sizes and which drives the length of the BDS system to about $3 \mathrm{~km}$. In addition, the BDS provides instrumentation to monitor key physics parameters, such as energy and polarisation. Due to the crossing angle of $20 \mathrm{mrad}$, crab cavities are required to rotate the bunches for head-on collisions.

The last part of the BDS consists of the final focus system. This system focuses the beams to the required sizes in order to reach the luminosity goals. It incorporates a chromaticity correction scheme [5], so that an energy spread in the beam will not dilute the beam size at the IP. The Final Doublet (FD) consists of the last two quadrupoles, named QF1 and QD0 (see fig. 5). The last quadrupole QD0 is located inside the detector. Given the beam sizes, colliding the beams at the IP imposes very tight tolerances on the stability of the FD quadrupoles.

\subsection{Dynamic imperfections}

\subsubsection{Ground motion}

GM induces beam misalignment and beam-beam offset leading to a loss of luminosity (luminosity is inversely proportional to the cross section of the bunches). The PSD of the natural GM is a steep function of frequency which falls 
off as $1 / f^{4}$. Several peaks can be observed and are related to machinery and structural resonances. Such peaks appear as steps in the IRMS. As the future CLIC location site is still unknown, different GM models based on measurements performed in accelerator laboratories and on historical data have been established [6]. Fig. 2 shows the PSD of the GM measured in the tunnel of the Large Hadron Collider (LHC) at CERN (where the Compact Muon Solenoid (CMS) [7] is located, report available in [8]), as well as several GM models. These GM models have been integrated in PLACET in order to characterize accurately the GM influence on the linear collider.

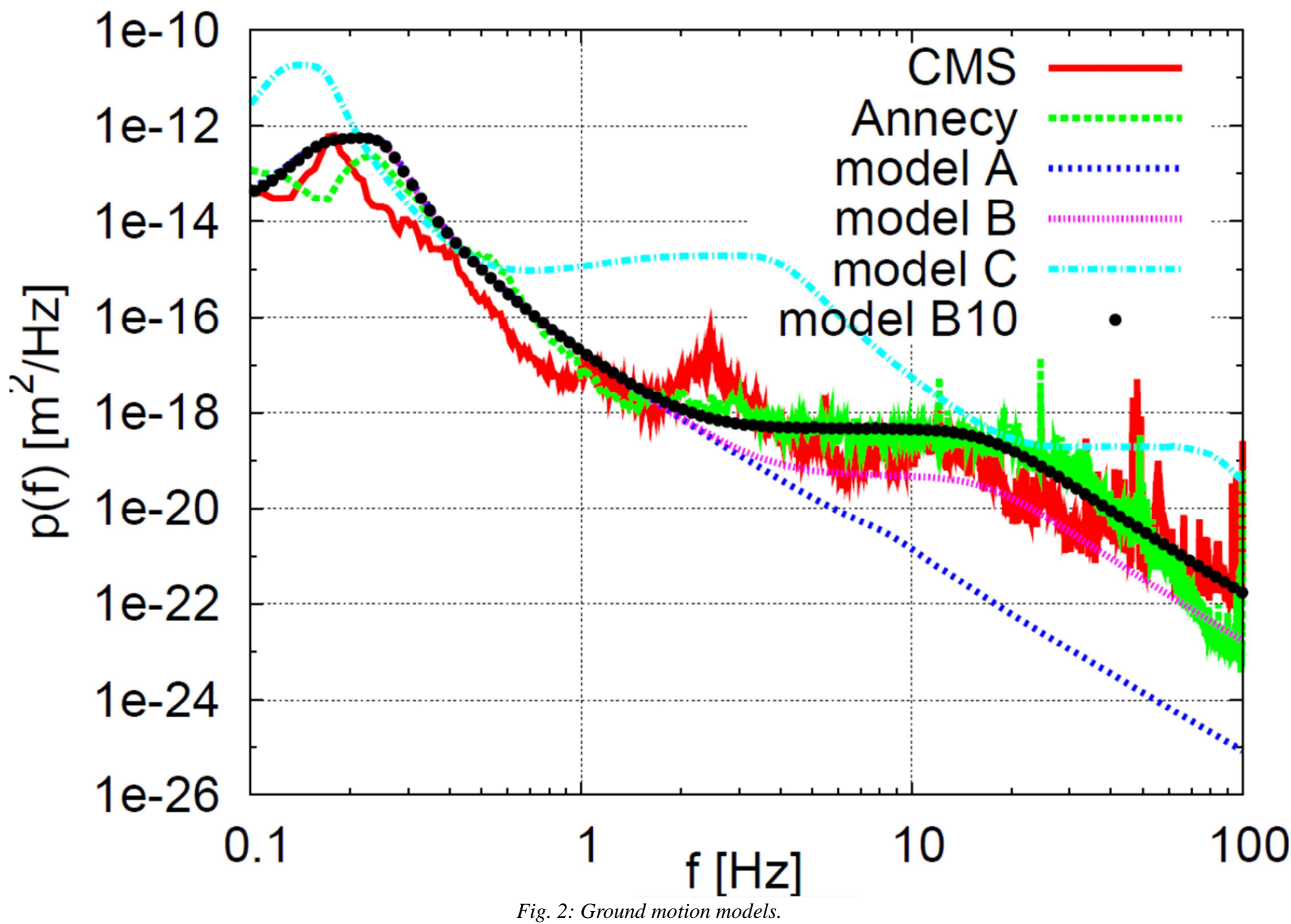

Model A is based on measurements in the empty LEP ${ }^{1}$ tunnel (no technical noise). Model B is made to fit measurements on the Fermilab ${ }^{2}$ site. Model B10 is made to fit measurements at LAPP in Annecy and the technical noise measured in the

\footnotetext{
${ }^{1}$ Large Electron Positron, (predecessor of the LHC, Geneva, Switzerland)

${ }^{2}$ Fermi National Accelerator Laboratory, (Batavia, Chicago).
} 
CMS hall. Finally, model $\mathrm{C}$ is based on measurements at DESY ${ }^{3}$.

\subsubsection{Direct disturbances on the quadrupole (Acoustic noise, cooling)}

Direct forces are transmitted through the beam pipe, power leads with cooling water pipes and by acoustic pressure, and act directly on the quadrupole [9] (see fig. 3).

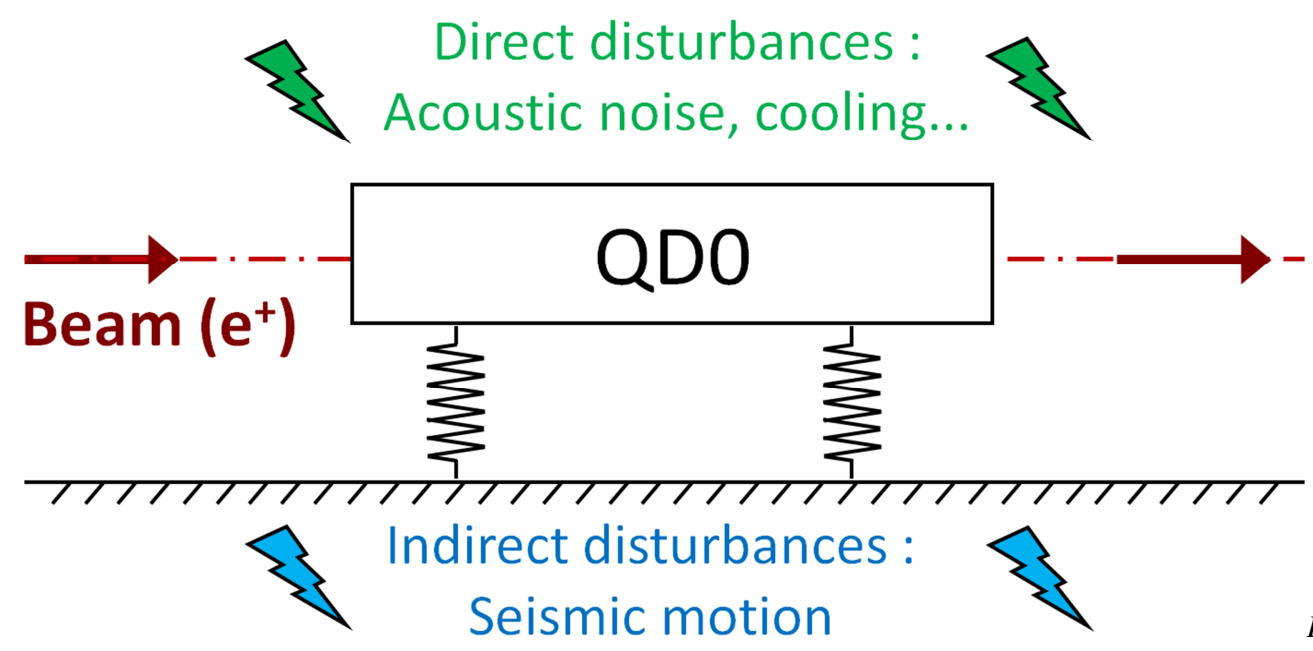

Fig. 3: Disturbances on the quadrupoles.

Recent studies [10], [11] have shown that the effect of the vibration forces acting directly on the magnet are highly attenuated by increasing the stiffness of the ML quadrupoles and its supports. The direct forces have been added in the GM model B10, which contains generic technical noise.

\subsection{Mechanical stabilisation systems}

\subsubsection{Mechanical stabilisation system for ML and BDS}

To reduce the motion of the ML quadrupoles for high frequencies ( $\geq 1 \mathrm{~Hz}$ ), each quadrupole will be positioned on an active stabilization system. Regarding the integrated simulations, a theoretical fit of the measured transfer functions of the current design [12] has been used, which is shown in fig. 4. An IRMS movement of $1 \mathrm{~nm}$ above $1 \mathrm{~Hz}$ has been demonstrated. The peak at $0.2 \mathrm{~Hz}$ of the Quadrupole Stabilization (QS) is close to the micro-seismic peak which is unfavourable. Thus, a targeted future design [13] is also shown in fig. 4. For the BDS, the same design as for the ML has been assumed in simulation, nevertheless, a more dedicated system could be envisaged.

\footnotetext{
${ }^{3}$ German Electron Synchrotron (Hamburg, Germany)
} 


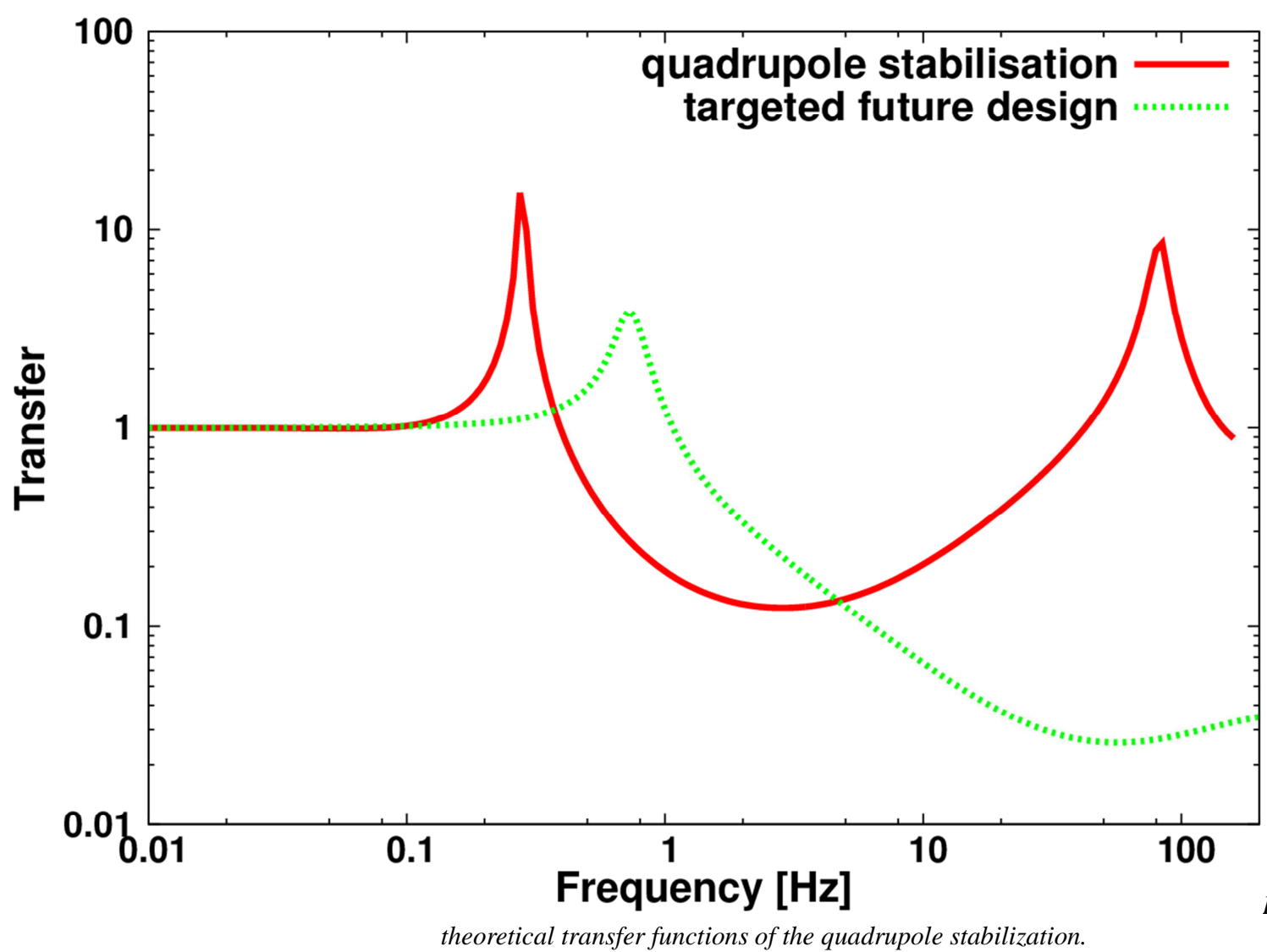

Fig. 4: Amplitude of the

\subsubsection{Mechanical stabilization system for the final doublet}

To reduce the beam offset jitter for high frequencies, the FD, which includes the last quadrupoles QD0 and QF1, will be put on a large mass, the pre-isolator [14] (see fig. 5), which is attached to the tunnel. In addition an active stabilization can be deployed, but the simulation is limited to the stand-alone usage of the pre-isolator. 


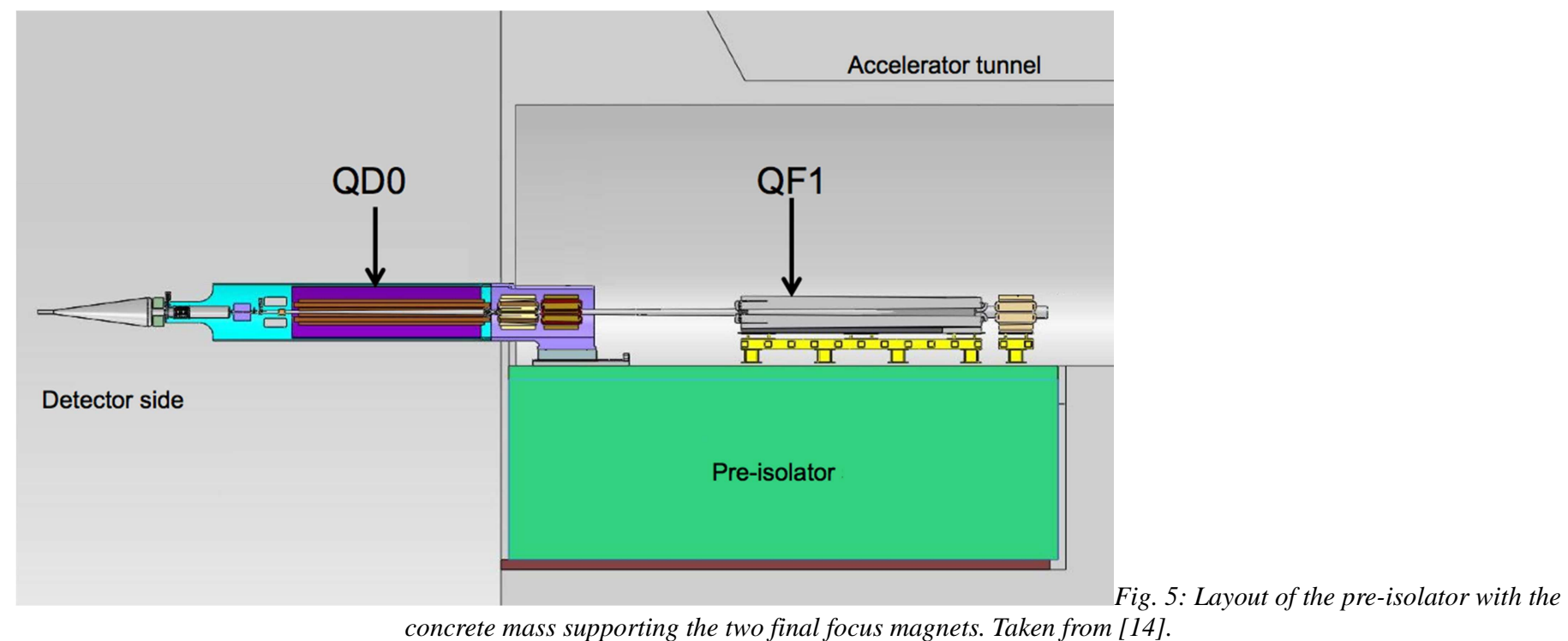

The two magnets are supported by rigid girders that are fixed on top of a massive concrete block, weighing about 80 tons and resting on several springs whose rigidity is tuned in order to have a vertical resonance of the whole assembly at $1 \mathrm{~Hz}$. For such a system, an achieved IRMS movement of $0.13 \mathrm{~nm}$ above $4 \mathrm{~Hz}$ has been reported [14]. The pre-isolator has two support points. Each of them has its own transfer function shown in fig. 6 . The resonance at $50 \mathrm{~Hz}$ is caused by the vibration of the cantilever and is designed to be at the beam repetition rate.

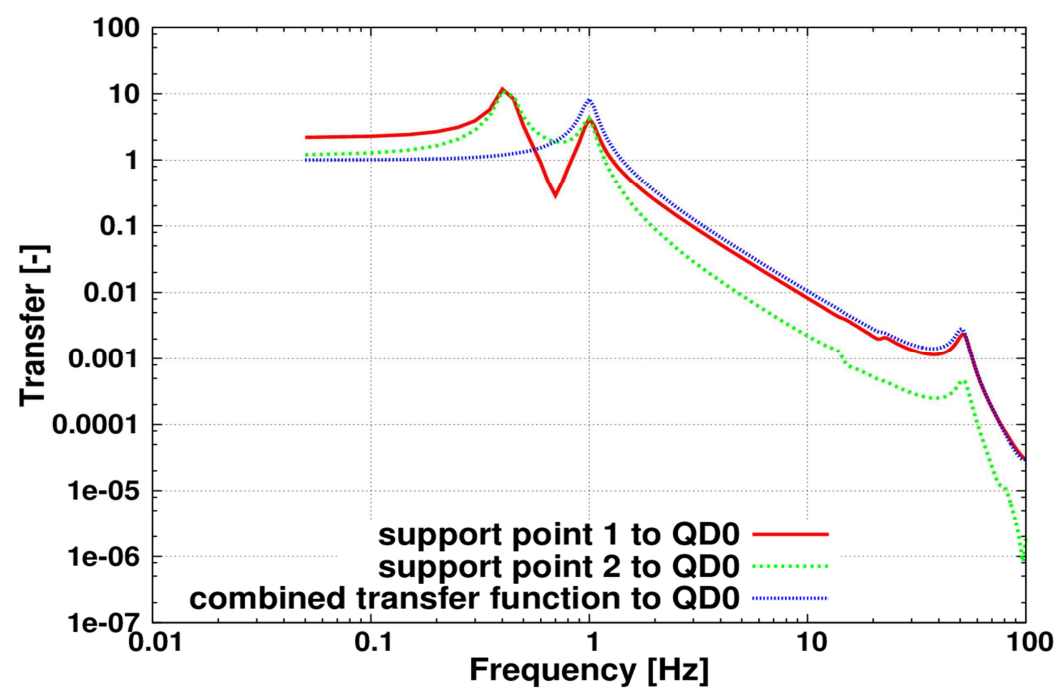

Fig. 6: Amplitude of the theoretical transfer functions of the pre-isolator of the final doublet system.

These transfer functions have been implemented in the simulation layout. A discrepancy between the two different 
transfer functions of the mechanical stabilization of the ML and BDS, and the final doublet system, and therefore between the quadrupole positions will impact luminosity. Therefore, the transfer functions should be tuned in order to have a similar shape, especially at lower frequencies. Simulations have shown that a tuning of the quadrupole stabilisation by e.g. a low-pass filter will ease the task of the OFB [13].

\section{Beam-based feedbacks}

Two main strategies are currently studied: the Interaction Point Feedback (IPFB) and the Orbit Feedback (OFB) are trajectory feedbacks, which collect information along the accelerator and at the interaction point in order to correct the beam positions thanks to specific kickers all along the accelerator. The sampling rate of these feedbacks is fixed and imposed by the beam repetition rate of $50 \mathrm{~Hz}$. This limitation causes these feedbacks to be only effective for frequencies below 1-4 Hz. The trajectory feedbacks are therefore only efficient at reducing beam size growth and slowly changing beam-beam offset.

\subsection{OFB control principle}

The OFB is intended to suppress GM, which induces beam oscillations along the ML and the BDS of CLIC. These beam oscillations degrade the luminosity performance, since they generate beam-beam offset and increase the emittance due to chromatic dilutions via filamentation. To counteract the beam oscillations, 2122 BPMs are installed along the ML and BDS, which measure the beam offsets in horizontal and vertical direction. These measurements are used by the OFB to calculate corrections that are sent to the actuators. The baseline choice for the actuators design is to move the quadrupoles mechanically with the help of the positioning capabilities of the stabilisation system presented in section 2.3.1. As an alternative solution, also dipole corrector magnets can be deployed.

The OFB is a discrete-time control system, with a sampling time of $20 \mathrm{~ms}$ (determined by the beam repetition rate). The design of the OFB is carried out in three steps. In the first step, the large, Multi-Input, Multi-Output (MIMO) accelerator system is decoupled, such that each input of the new system is only influencing one output. This is accomplished by transforming the inputs and outputs by matrix multiplications that can be found with the Singular Value Decomposition (SVD) of the orbit response matrix. With this SVD decoupling, the design is simplified significantly, since the task of 
finding one large MIMO controller is split up into the determination of simpler Single-Input Single-Output controllers (SISO). For more detailed information on the OFB, refer to [15].

In the second and third steps of the design, the individual SISO controllers are determined. The second step consists of the determination of one controller transfer function $C(z)$, which shapes the form of all open loop frequency responses of the decoupled accelerator channels in an appropriate way. This shaping ensures high GM suppression at low frequencies, low noise amplification at high frequencies and takes into account stability consideration by guaranteeing a phase margin of more than $36^{\circ}$. In the third step, one open gain parameter $g_{i}$ is calculated per decoupled accelerator channel, which is multiplied with the before defined controller $C(z)$ to get the final SISO controllers $g_{i} C(z)$, where $i$ is the channel index. The parameter $g_{i}$ is determined such that the luminosity loss due to GM and measurement noise is minimised, by balancing the two influences. For this balancing, models of GM and measurement noise as well as a model of the effect of these signals on the luminosity loss are used. fig. 7 shows closed loop frequency responses magnitudes of the beam oscillation amplitude to GM ( $\left.T_{g a}\right)$ and to measurement noise $\left(T_{n a}\right)$, for one decoupled control loop.

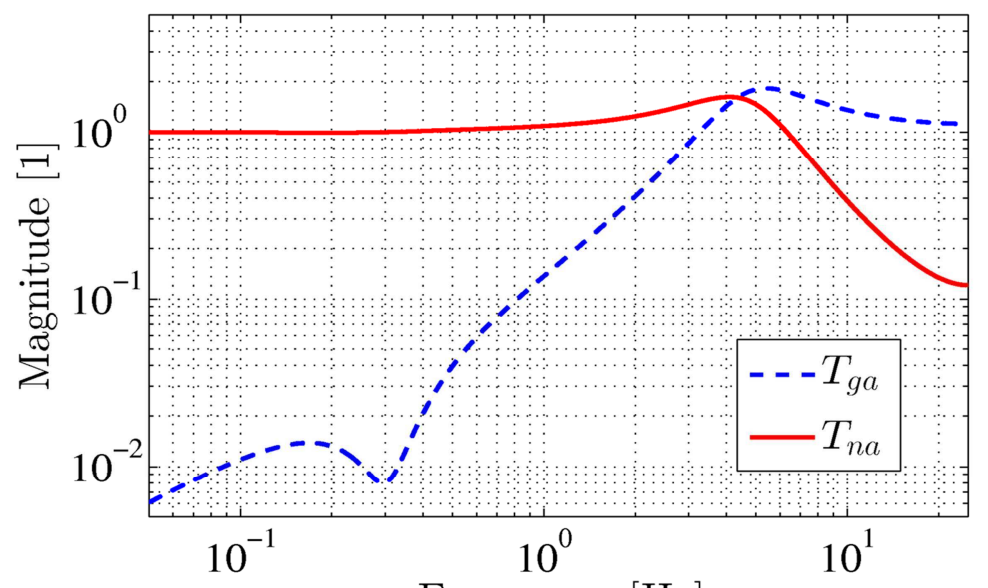

Frequency $[\mathrm{Hz}]$

Fig. 7: Magnitudes of the closed loop frequency responses of the beam oscillation amplitude of one decoupled control loop to ground motion $\left(T_{g a}\right)$ and measurement noise $\left(T_{n a}\right)$. For this plot, a $g_{i}$ of 1 is assumed.

\subsection{IPFB control principle}

The IPFB corrects the beam position at the IP $\delta_{y}$ by measuring the deflection angles of the colliding beams and adjusting the beam position with a dipole kicker positioned near QD0. The structure of this control is given in fig. 8. 
The proposed control framework is composed of a feedback loop with a numerical controller $(H)$ optimized to minimize the offset of the beam, and a real-time adaptive control feature $\left(H_{a}\right)$ based on the Generalized Least Squares (GLS) algorithm [16]. A more detailed study of the whole control can be found in [17].

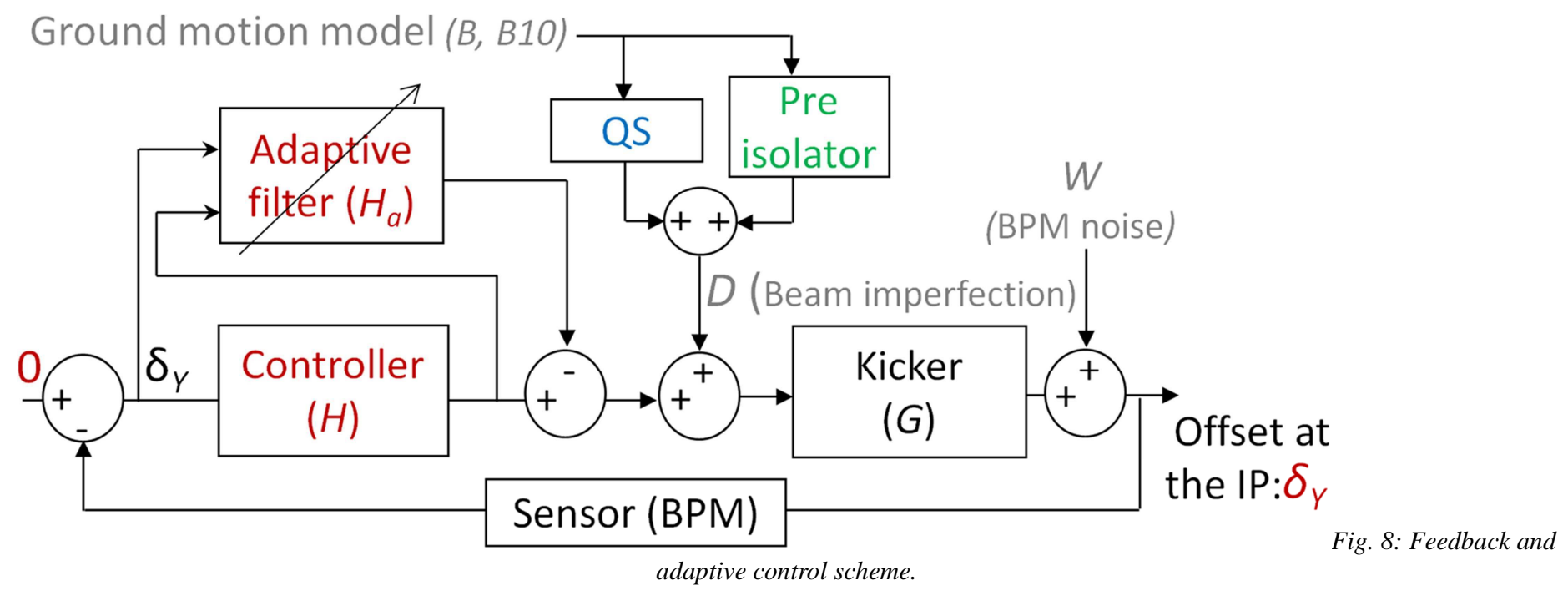

The disturbance $(B, B 10)$ is the mechanical excitation from GM. $(D)$ is the disturbance felt by the quadrupole leading to beam imperfections (i.e. GM models damped by the mechanical stabilizations: QS or pre-isolator). The transfer function between the mechanical displacement of this quadrupole and the beam can be modelled by a constant gain equal to 1 since it is considered as a uniform rigid structure. The transfer function of the BPM is also modelled by a unity gain, since the beam offset $\delta_{y}$ feeds the input of the controllers.

The action, meant to reduce the offset between the two beams at the IP, is done by a kicker. The obtained displacement of the beam is proportional (equal to 1 in the following model) to the injected current of the kicker. The dynamic of the process is a delay at a sampling period equal to $0.02 \mathrm{~s}$.

The structure of the compensator $(H)$ is given by $H(q)=\frac{b_{0}+b_{1} q^{-1}+b_{2} q^{-2}}{1+a_{1} q^{-1}+a_{2} q^{-2}}$ where $q^{-1}$ is the back shift operator.

In the following simulations, five control strategies have been tested: 
a particular

case where $a_{1}$ and $a_{2}$ are equal to -1 without adaptive filter, that corresponds to a double integrator in the controller, called PIID,

a particular

case where only $a_{1}$ is equal to -1 without adaptive filter, that corresponds to a single integrator in the controller, called PID,

a general

case where $a_{1}$ nor $a_{2}$ are imposed without adaptive filter, called FB,

a particular

case where only $a_{1}$ is equal to -1 with the adaptive filter, called PIDA,

a general

case where $a_{1}$ nor $a_{2}$ are imposed with the adaptive filter, called FBA (see [17] for more details).

\section{$4 \quad$ Simulation and results}

\subsection{Simulation setup}

Simulations of the beam dynamics in CLIC, from the entrance of the ML to the IP including the beam-beam interaction have been performed by using PLACET and GUINEA-PIG. All mitigation techniques have been implemented. The measured PSDs and the luminosity have been averaged over 100 seeds each corresponding to a real-time accelerator operation of $15 \mathrm{~s}$ for GM B and B10.

\subsection{Modelling assumptions}

Main assumptions leading to the results presented in this paper such as hardware dynamic response or BPM resolution are discussed in this section. First of all, the transmittance of the quadrupole has been neglected, which corresponds to an infinite rigid quadrupole. Although this isn't the case, the stiff design of the quadrupole would prevent any resonant frequency below several hundred Hz. Moreover, the GM PSD decreases rapidly with frequency being proportional with $1 / f^{4}$, and the mechanical stabilisation should damp any vibration above a few Hz. Because of these considerations, the dynamic response of the quadrupole should have a negligible impact on the results. Furthermore, in simulation each quadrupole has the same transfer function, while in reality there will be a certain spread in them, which will degrade the performance of the OFB and might result in more beam jitter at the IP. This will be a topic for future study. Next, the 
kicker's dynamic response has been neglected, since it is assumed to be much faster than the sampling period of the beam. This is a specification requirement for the development of such an actuator, and should be possible to achieve regarding the low beam repetition rate of $50 \mathrm{~Hz}$. Finally, in these simulations a resolution of 50/100 $\mathrm{nm}$ [18] has been used for BDS/ML BPMs, while the post-collision BPM resolution has been neglected. This will have a clear impact on the results. Fig. 9 shows the evolution of the luminosity loss and the beam-beam offset IRMS @ $0.1 \mathrm{~Hz}$ as a function of the postcollision BPM resolution.

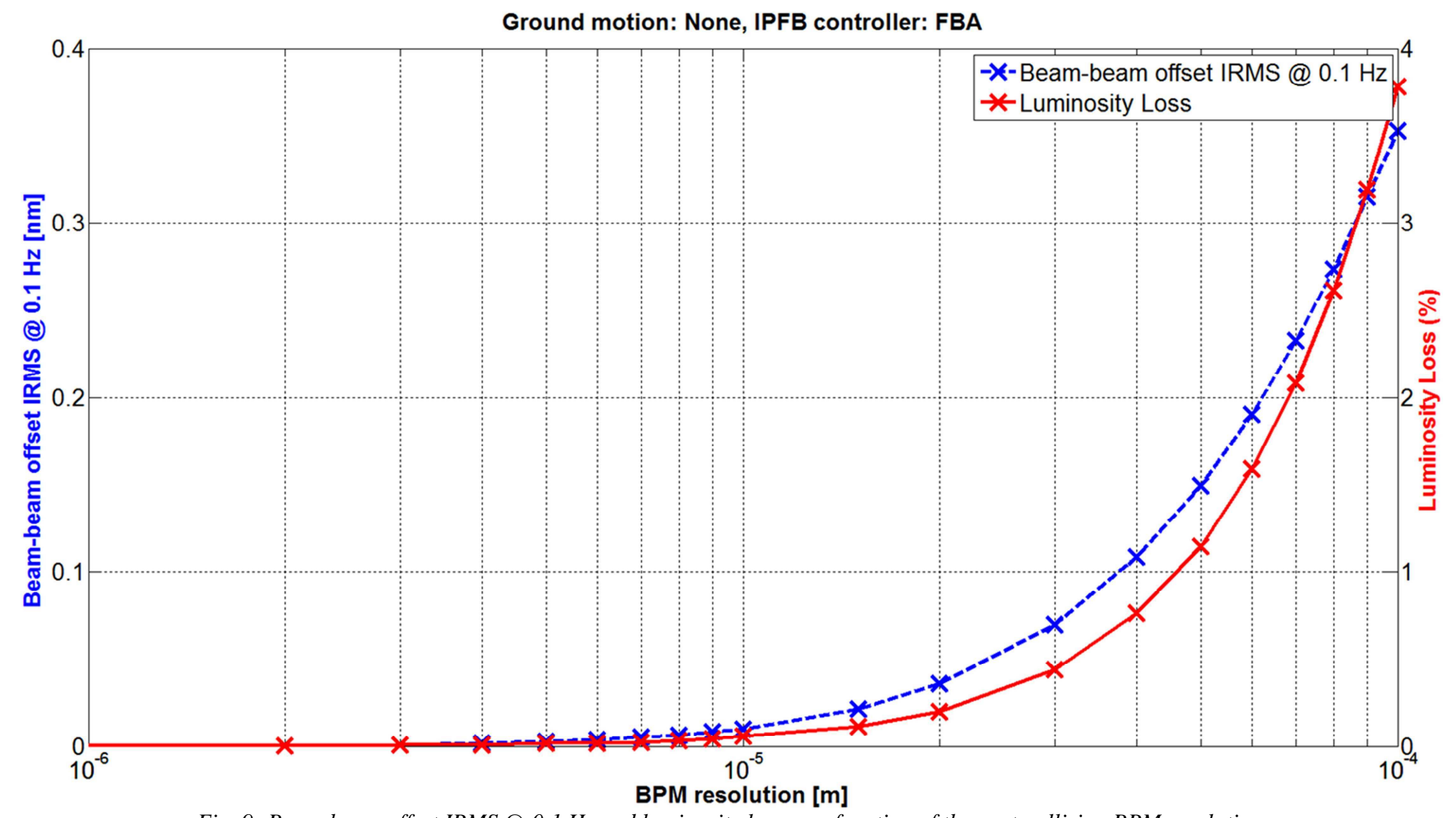

Fig. 9: Beam-beam offset IRMS @ $0.1 \mathrm{~Hz}$ and luminosity loss as a function of the post-collision BPM resolution.

These results have been obtained with the FBA controller and without GM. The plot shows the correlation between the beam-beam offset and the luminosity loss. The latter is limited to $1 \%$ considering a resolution of about $45 \mu \mathrm{m}$ for the post-collision BPM. This simulation can be considered realistic enough to specify the post-collision BPM resolution.

\subsection{Feedback simulations}

The first set of simulations shows on the left part of fig. 10 the beam-beam offset PSD and IRMS when using the five different IPFBs discussed in section 3.2 with GM model B10. The right part shows the same results with GM model B. The two feedbacks (IPFB and OFB) are on. 

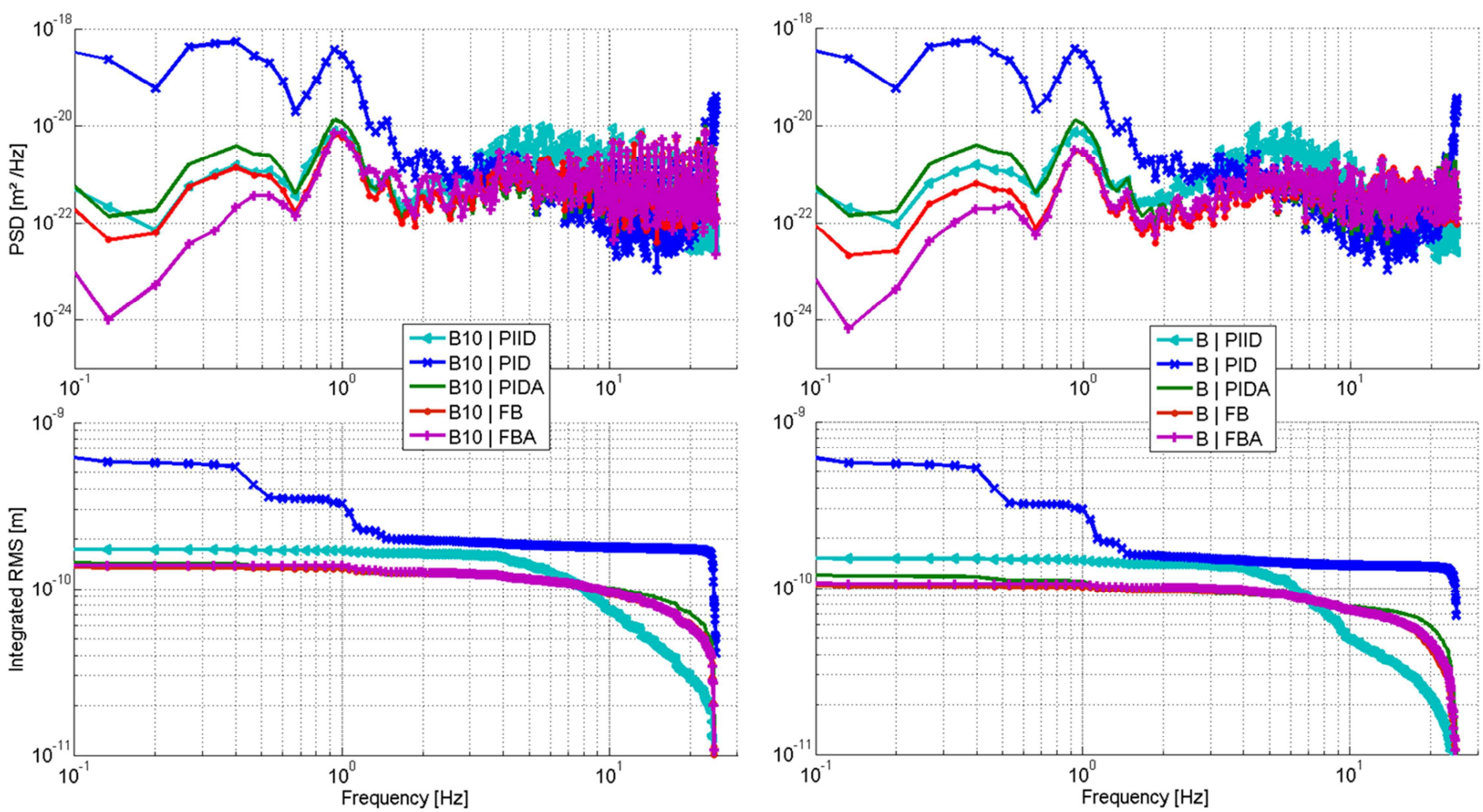

Fig. 10: IP Feedback controller comparison for GM B10 and B with both BBFBs.

Tables 1 and 2 summarise the obtained performances in terms of luminosity loss and IRMS @ $0.1 \mathrm{~Hz}$. Two other GM models A and C (see fig. 2) have been added for comparison.

Table 1

Average luminosity loss.

\begin{tabular}{|c|l|c|cccc|}
\hline \multicolumn{7}{|c|}{ Average luminosity loss, (SEM) } \\
\hline \multicolumn{2}{|c|}{ IPFB } & PIID & PID & PIDA & FB & FBA \\
\hline \multirow{3}{*}{$\begin{array}{c}\text { Ground } \\
\text { motion }\end{array}$} & $\mathrm{A}$ & $1.47 \%(0.01 \%)$ & $3.30 \%(0.06 \%)$ & $2.78 \%(0.05 \%)$ & $1.21 \%(0.01 \%)$ & $1.22 \%(0.01 \%)$ \\
\cline { 2 - 7 } & $\mathrm{B}$ & $1.63 \%(0.01 \%)$ & $7.10 \%(0.16 \%)$ & $1.54 \%(0.03 \%)$ & $1.47 \%(0.03 \%)$ & $1.49 \%(0.03 \%)$ \\
\cline { 2 - 7 } & $\mathrm{B} 10$ & $1.88 \%(0.01 \%)$ & $7.22 \%(0.14 \%)$ & $1.75 \%(0.03 \%)$ & $1.72 \%(0.03 \%)$ & $1.72 \%(0.03 \%)$ \\
\cline { 2 - 7 } & $\mathrm{C}$ & $40.98 \%(0.40 \%)$ & $47.40 \%(0.34 \%)$ & $27.38 \%(0.30 \%)$ & $22.76 \%(0.24 \%)$ & $22.02 \%(0.25 \%)$ \\
\hline
\end{tabular}

Table 2

IRMS of Beam-Beam offset for several GMs and IPFB controllers.

\begin{tabular}{|c|l|ccccc|}
\hline \multicolumn{7}{|c|}{ Beam-beam offset IRMS @ 0.1 Hz [nm], (SEM) } \\
\hline \multicolumn{2}{|c|}{ IPFB } & PIID & PID & PIDA & FB & FBA \\
\hline \multirow{2}{*}{$\begin{array}{c}\text { Ground } \\
\text { motion }\end{array}$} & $\mathrm{A}$ & $1.5 \mathrm{E}-10$ & $3.5 \mathrm{E}-10$ & $2.8 \mathrm{E}-10$ & $9.5 \mathrm{E}-11$ & $9.8 \mathrm{E}-11$ \\
& & $(1.1 \mathrm{E}-12)$ & $(7.9 \mathrm{E}-12)$ & $(5.4 \mathrm{E}-12)$ & $(3.9 \mathrm{E}-13)$ & $(3.9 \mathrm{E}-13)$ \\
\cline { 2 - 7 } & $\mathrm{B}$ & $1.6 \mathrm{E}-10$ & $6.3 \mathrm{E}-10$ & $1.2 \mathrm{E}-10$ & $1.1 \mathrm{E}-10$ & $1.1 \mathrm{E}-010$, \\
\hline
\end{tabular}


G. Balik, B. Caron, D. Schulte, J. Snuverink, J. Pfingstner

\begin{tabular}{|l|ccccc|} 
& $(9.9 E-13)$ & $(1.9 E-11)$ & $(7.7 E-13)$ & $(4.2 E-13)$ & $(1.0 E-012)$ \\
\hline \multirow{2}{*}{ B10 } & $1.9 \mathrm{E}-10$ & $6.4 \mathrm{E}-10$ & $1.5 \mathrm{E}-10$ & $1.5 \mathrm{E}-10$ & $1.5 \mathrm{E}-10$ \\
& $(1.2 E-12)$ & $(1.4 E-11)$ & $(8.7 E-13)$ & $(7.1 E-13)$ & $(8.2 E-13)$ \\
\hline \multirow{2}{*}{$\mathrm{C}$} & $2.3 \mathrm{E}-09$ & $2.6 \mathrm{E}-09$ & $9.2 \mathrm{E}-10$ & $5.9 \mathrm{E}-10$ & $4.5 \mathrm{E}-10$ \\
& $(4.3 E-11)$ & $(3.1 E-11)$ & $(1.3 E-11)$ & $(4.7 E-12)$ & $(3.3 E-12)$ \\
\hline
\end{tabular}

Statistical errors on the simulation results have been characterized by the Standard Error on the Mean (SEM) in the tables, which is given by the sample standard deviation divided by the square root of the number of seeds. These simulations show that the GM model has an important influence on the performances in terms of luminosity or offset. For example, with GM model C, the specifications cannot be reached with any of the controllers. In most cases, a simple PID controller isn't able to maintain good performances. At this moment, it is not possible to choose a particular controller due to some imperfections not taken into account in the simulation model and due to some future changes in the design of the components such as the pre-isolator. Nevertheless, the most important unknown is the characteristic of the GM that has an impact on the performances. The partial conclusion is that the proposed set of controller strategies can be used on a variety of reasonable GM models.

\subsection{Interaction between Feedbacks}

In section 4.3 all simulations were done with both feedbacks on and it is shown that the luminosity loss is sufficiently low compared to other imperfections when the GM model is not too severe. In these simulations, the coupling effects between the two feedback loops are investigated. The left part of fig. 11 shows the PSD and IRMS of the beam-beam offset for ground model B10 for the FBA controller and the right part shows the same results for ground model B. 

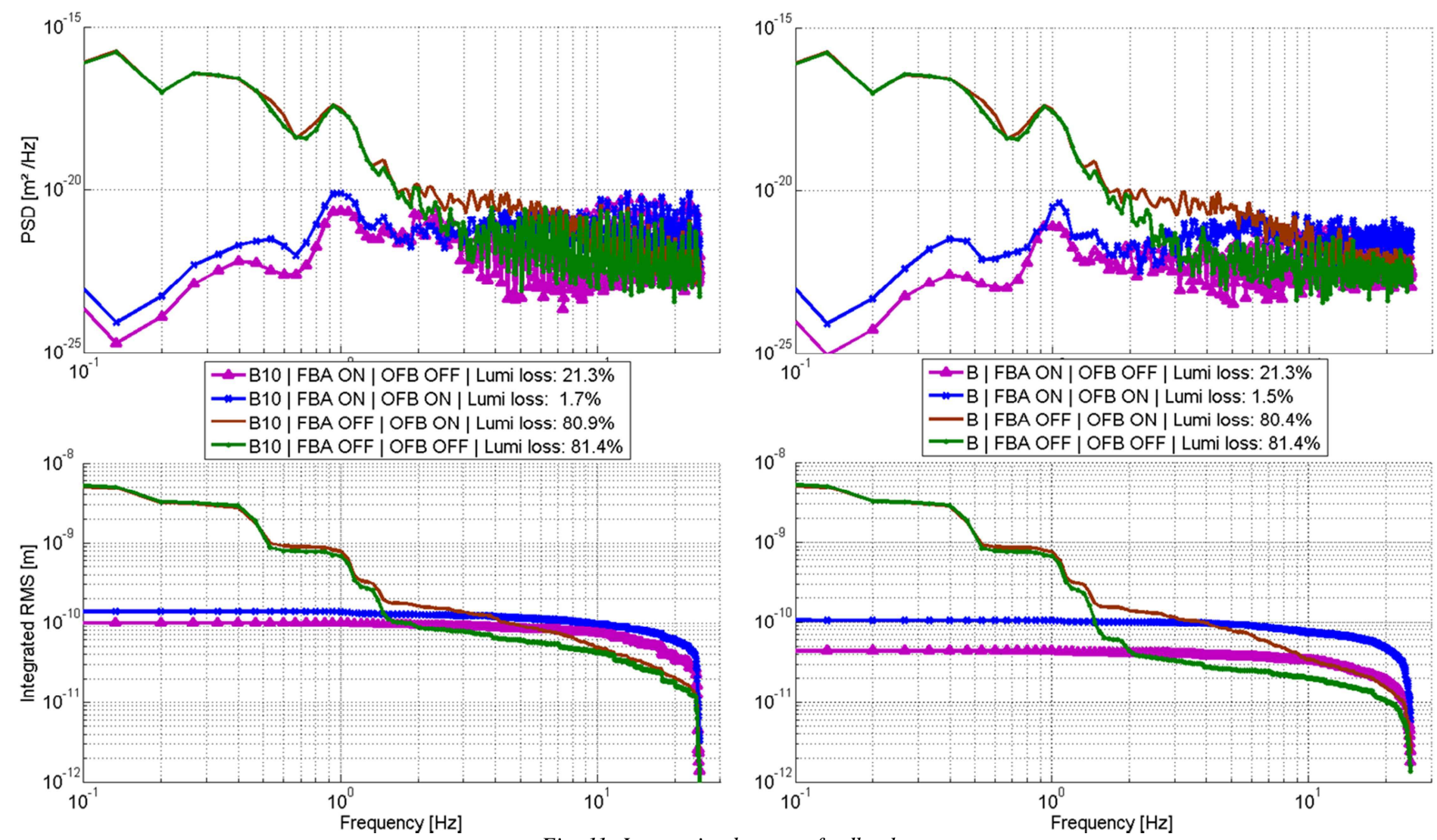

Fig. 11: Interaction between feedbacks.

These figures show that, in order to limit the luminosity loss, both feedbacks must be on. When only the OFB is on, both luminosity and beam-beam offset are far from the needed performances. With only the IPFB on, the IRMS is the lowest but without the needed luminosity. In any case where the IPFB is on, the GM is successfully corrected below $4 \mathrm{~Hz}$. The OFB has a small effect on the degradation of the beam-beam offset when both feedbacks are on, leading to an IRMS twice as high as without OFB. Simulations using other controllers show the same behaviour. Nevertheless, this degradation due to the introduction of the OFB is small enough and the beam-beam offset is under the needed maximum requirements. This scheme allows to reach an IRMS of the beam motion of about $0.2 \mathrm{~nm} @ 0.1 \mathrm{~Hz}$ with both feedbacks leading to an average luminosity loss of less than $1.7 \%$ (see table 1 ).

\section{Conclusion}

In this paper, an integrated simulation of GM mitigation techniques for the future compact linear collider has been shown. The simulation takes into account most of the physical elements of the collider: beam optics, mechanical supports, realistic models of GM, etc. Two feedbacks are presented; on the one hand, the IPFB meant to reduce the offset position 
of the colliding beams at the IP, on the other hand, the OFB designed to minimize the emittance of the beam. Both feedbacks aim to maximize the luminosity performance. For the IPFB five different control strategies are discussed. Different structures for the beam-beam offset control show interesting results. First, the presented controllers are able to deal with different GM behaviour except for high frequency disturbed models. Second, detrimental coupling between both feedbacks have been shown to exist and it increases the beam-beam offset up to a factor two. However, these controllers can maintain the beam-beam offset around $0.2 \mathrm{~nm}$ (IRMS @ $0.1 \mathrm{~Hz}$ ), resulting in less than $1.7 \%$ average luminosity loss for both considered GM models. Overall, with the various adaptable IPFB and OFB controllers and the well-advanced integrated simulation, we believe that we are flexible to choose the correct controllers to tackle the unknown, to be encountered GM successfully. Finally, the obtained performances are above the nominal performances needed for the collider as the $1.7 \%$ is deemed to be small enough compared to the $20 \%$ luminosity loss budget for all dynamic effects in the accelerator. The quoted luminosity losses are due to the GM and controllers directly, while some simplifications have been made in the simulation, which will degrade the luminosity performances. Specifically, the simplifications concerning the transfer functions and the BPM resolution are discussed in detail. A further improvement can be made with a global optimisation of both FBs' parameters and mechanical stabilisation systems in order to increase the performances and robustness of the presented strategy with respect to non-modelled behaviour of the collider.

\section{Acknowledgement}

The research leading to these results has received funding from the European Commission under the FP7 Research Infrastructures project EuCARD, grant agreement no.227579. The authors wish to express their thanks to A. Jeremie, for this project and fruitful collaboration.

\section{Nomenclature Tables}

$\begin{array}{ll}\text { BBFB } & \text { Beam-Based FeedBack } \\ \text { BDS } & \text { Beam Delivery System } \\ \text { BPM } & \text { Beam Position Monitor } \\ \text { CERN } & \text { European Organization for Nuclear Research } \\ \text { CLIC } & \text { Compact Linear Collider } \\ \text { CMS } & \text { Compact Muon Solenoid } \\ \text { FD } & \text { Final Doublet }\end{array}$




$\begin{array}{ll}\text { GLS } & \text { Generalized Least-Square } \\ \text { GM } & \text { Ground Motion } \\ \text { IP } & \text { Interaction Point } \\ \text { IPFB } & \text { Interaction Point FeedBack } \\ \text { IRMS } & \text { Integrated Root Mean Square } \\ \text { LAPP } & \text { Laboratoire d'Annecy-Le-Vieux de Physique des Particules } \\ \text { LEP } & \text { Large Electron Positron } \\ \text { LHC } & \text { Large Hadron Collider } \\ \text { MIMO } & \text { Multiple Input Multiple Output } \\ \text { ML } & \text { Main Linac } \\ \text { PSD } & \text { Power Spectral Density } \\ \text { OFB } & \text { Orbit FeedBack } \\ \text { QD0/QF1 } & \text { Final Doublet Quadrupoles } \\ \text { QS } & \text { Quadrupole Stabilization } \\ \text { RF } & \text { Radio Frequency } \\ \text { SEM } & \text { Standard Error of the Mean } \\ \text { SISO } & \text { Single Input Single Output } \\ \text { SVD } & \text { Singular Value Decomposition }\end{array}$
$A, B, B 10, C$
Ground motion models
$C$
SISO OFB controller
$D$
Beam imperfection

$F B(A), P I D(A)$,

(Adaptive) Numerical and Proportional-Integral-Derivative controllers

$G$

Kicker (actuator used to steer the beam)

$H$

Numerical controller

$H_{a}$

Adaptive filter

$L_{(0)}$

$T_{g a}, T_{n a}$

(Nominal) luminosity

Closed loop frequency responses magnitudes of the beam oscillation amplitude to $\mathrm{GM}\left(\mathrm{T}_{\mathrm{ga}}\right)$ and to measurement noise $\left(\mathrm{T}_{\mathrm{na}}\right)$

$\sigma_{x, 0} \sigma_{y, 0}$

(Nominal) beam size at the IP along $\mathrm{x}$ and $\mathrm{y}$

$\delta$

Beam-beam offset

$g_{i}$

Gain parameter corresponding to channel index i

$x, y$

Horizontal and vertical direction

\section{References}

[1] CLIC collaboration, CLIC conceptual design report, CERN, 2012.

[2] D. Schulte, PLACET - A program to simulate drive beams, Proceedings of European Particle Accelerator Conference (EPAC'00), Vienna, Austria, 2000. 
[3] D. Schulte, Beam-beam simulations with GUINEA-PIG, ICAP'98, Monterey, CA., USA, 1998, 1998.

[4] D. Schulte, Emittance Preservation in the Main Linac of CLIC, Proceedings of European Particle Accelerator Conference (EPAC'98), Stockholm, Sweden, 1998.

[5] R. Tomas et al., Status of the CLIC beam delivery system, Particle Accelerator Conference (PAC'09), Vancouver, Canada, 2009.

[6] A. Seryi, Ground Motion Models for Future Linear Colliders, Proceedings of European Particle Accelerator Conference (EPAC'00), Vienna, Austria, 2000.

[7] The CMS Collaboration, The CMS experiment at the CERN LHC, Journal of Instrumentation 3S08004 26-89, 2008.

[8] A. Kuzmin et al., Ground vibration measurements and experiment parts motion measurement at CMS, Internal note, CERN document server, EDMS Nr: 1027459, 2009.

[9] B. Bolzon, Etude des vibrations et de la stabilisation à l'échelle sous-nanométrique des doublets finaux d'un collisionneur linéaire, Thesis, LAPP-T-2007-05, Université de Savoie (France), 2006.

[10] K. Artoos et al., Modal analysis and measurement of water cooling induced vibrations on a CLIC main beam quadrupole prototype., Proceedings of International Particle Accelerator Conference (IPAC'11), MOPO028, San Sebastian, Spain, 2011.

[11] B. Bolzon, J.P. Baud, G. Gaillard, N. Geffroy, A. Jeremie, Impact of flowing cooling water on the ATF2 final, ATF report, ATF $09-01,2009$ / 04 / 1, 2009.

[12] K. Artoos et al., Status of a study of stabilization and fine positioning of CLIC quadrupole to the nanometer level, Proceedings of International Particle Accelerator Conference (IPAC'11), MOPO027, San Sebastian, Spain, 2011.

[13] S. Janssens et al., System control for the CLIC main beam quadrupole stabilization andnano-positioning, Proceedings of International Particle Accelerator Conference (IPAC'11), TUPC014, San Sebastian, Spain, 2011.

[14] A. Gaddi, H. Gerwig, N. Siegrist, F. Ramos, Dynamic analysis of the FF magnets pre-isolator and support system, EDMS Document Server, EDMS n $1098581,2010$.

[15] J. Pfingstner et al., SVD-based filter design for the trajectory feedback of CLIC, Proceedings of International Particle Accelerator Conference (IPAC'11), MOPO014, San Sebastian, Spain, 2011.

[16] I.D. Landau and G. Zito, Digital control systems: Design, identification and implementation, Communications and control engineering. Springer Verlag, 2006.

[17] B. Caron, G. Balik, L. Brunetti, A. Jeremie, Adaptive vibration control of the beam of the future linear collider, Control Engineering Practice, 2011.

[18] J. Snuverink et al., Status of ground motion mitigation techniques for CLIC, Proceedings of International Particle Accelerator Conference (IPAC'11), TUPC023, San Sebastian, Spain, 2011. 